\title{
Machine Learning Assisted Pattern Matching: Insight into Oxide Electronic Device Performance by Phase Determination in 4D-STEM Datasets
}

Alexander Zintler ${ }^{1}$, Robert Eilhardt ${ }^{1}$, Shuai Wang ${ }^{1}$, Matus Krajnak ${ }^{2}$, Patrick Schramowski ${ }^{1}$, Wolfgang Stammer $^{1}$, Stefan Petzold ${ }^{1}$, Nico Kaiser ${ }^{1}$, Kristian Kersting ${ }^{1}$, Lambert Alff ${ }^{1}$ and Leopoldo Molina-Luna ${ }^{1}$

${ }^{1}$ Technical University of Darmstadt, Darmstadt, Hessen, Germany, ${ }^{2}$ Quanum Detectors Ltd., Harwell Oxford, England, United Kingdom

Improving device reliability and performance in oxide electronic-based resistive memory applications requires a profound understanding of the microstructure and atomistic processes which define the device properties. Here, we investigated a molecular beam epitaxy (MBE) grown thin film stack composed of a $10 \mathrm{~nm} \mathrm{HfO} 2$ layer deposited on a (111) oriented TiN bottom electrode. The resulting $\mathrm{HfO}_{2}$ grains grow in a highly textured manner with their (11-1) axis oriented out of plane. A set of only 6 in-plane-rotations is allowed for the $\mathrm{HfO}_{2}$ grains, which limits the number of possible grain boundary geometries [1]. These grain boundaries have a strong influence on the macroscopic device behavior due to their inherent physical properties, i.e. localized high defect concentration [2] and increased vacancy mobility [3]. The direct impact of such nanoscale inhomogeneities on the device properties can be controlled via (i) oxygen engineering, (ii) grain-boundary engineering and (iii) by using more complex stack geometries.

While 4D-STEM methodologies can resolve the relevant structural features [4], acquiring statistical information is a time-consuming process. Here, we present a procedure that allows for fast acquisition of spatially correlated, multi-phase information in a two (or more) layered thin film stack. By transmitting a precessing quasi-parallel STEM beam through the sample (see Fig. 1a, also referenced as Scanning Precession Electron Diffraction, SPED) a four-dimensional data set is generated. In our setup, scanning of an NBD probe (in 2D real space) was controlled by using a NanoMEGAS P1000 scanning unit integrated into a JEOL-ARM200F. A fast and high dynamic range Medipix3 detector (Merlin, Quantum Detectors) was used for synchronized diffraction pattern acquisition (in 2D reciprocal space).

The stack was FIB prepared in plan-view geometry which allows the direct correlation of the epitaxial texture transfer for each individual $\mathrm{HfO}_{2}$ grain over a large sample area. The result is a set of superimposed diffraction patterns of all sampled phases contained in the stack (see figure 1b). In order to distinguish between phases, we applied both, pattern matching (PM) and machine learning (ML) based blind source separation (BSS) routines [5]. Conventional PM discriminates the phase and orientation by solving each pattern for the best match in a simulated list of templates, however, this approach yields a low reliability phase recognition for a mixed diffraction pattern. Using a list of components generated by unsupervised single value decomposition (SVD), the raw data were filtered (see figure 2). This yields an improved PM result. Recent developments in ML mainly contributed to the advancements in deep learning, have brought large improvements to interdisciplinary fields, e.g. signal processing and microscopy [6]. Deep neural networks (DNN) therefore present a promising extension to the ML based BSS routines. Deconvolving multi-phase superimposed SPED datasets, as presented here, can offer a unique and statistical way of analyzing complex crystalline thin film stacks with a high spatial resolution [7]. 

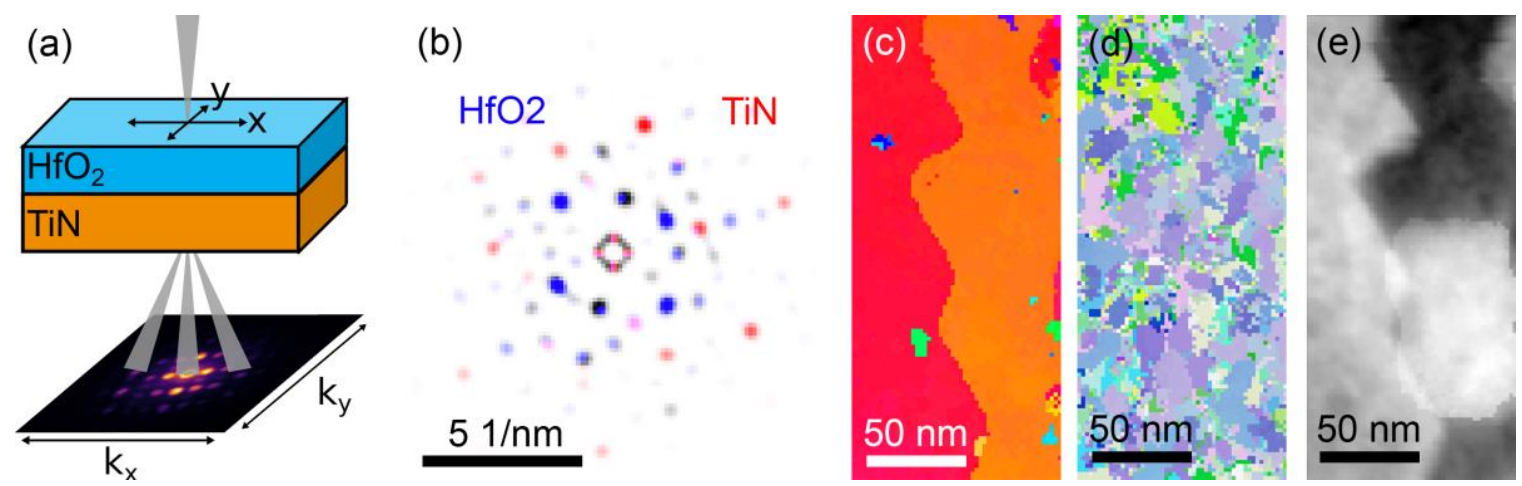

Figure 1. (a) Quasi-parallel 4D-STEM (SPED) and plan-view sample geometry, (b) single color-coded superimposed diffraction pattern of a HfO2 (blue) and a TiN grain (red), (c) orientation map (IPF colorcoding) of the TiN phase and (d) of the HfO2 phase for the same area, (e) exemplary extracted SVD loading revealing one of the TiN grain orientations. The component also reveals amorphous carbon contamination.

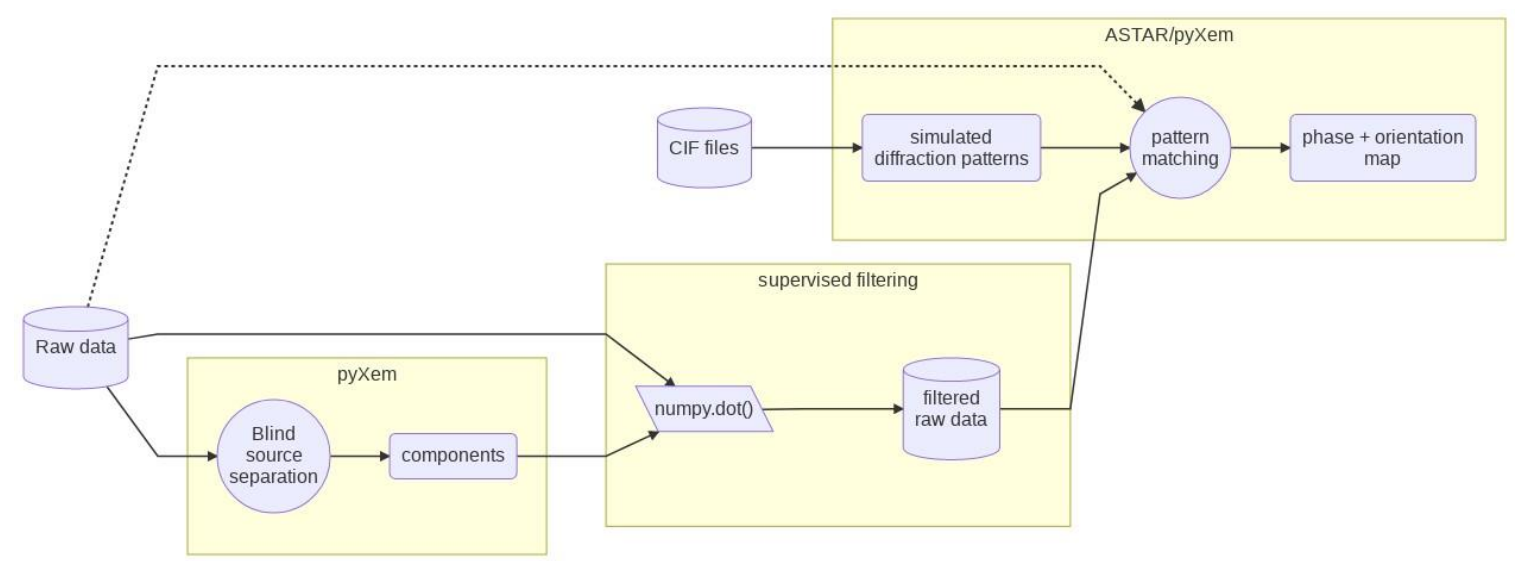

Figure 2. Flow-chart of the suggested data processing routine.

\section{References}

[1] Petzold S, Zintler A, et al., Adv. Electron. Mater. 10 (2019) 10.1002/aelm.201900484

[2] Xue K-H, Blaise P, et al., Appl. Phys. Lett. 20 (2013) 10.1063/1.4807666

[3] McKenna K, Shluger A, Appl. Phys. Lett. 22 (2009) 10.1063/1.3271184

[4] Ophus C, Microsc. Microanal. 3 (2019) 10.1017/S1431927619000497

[5] Duncan N. Johnstone, Phillip Crout, et al., (2020) 10.5281/zenodo.3667613

[6] Borodinov, N., Neumayer, et al. npj Comput Mater 5, 25 (2019). 10.1038/s41524-019-0148-5

[7] The authors acknowledge financial support from the Deutsche Forschungsgemeinschaft (DFG) under research grant MO 3010/3-1 and the European Research Council (ERC) "Horizon 2020" Program under Grant No. 805359-FOXON. 